\title{
Uncertainty Evaluation on Determination of Aurum in E-waste by ICP-AES
}

\author{
Wang Yong-Le ${ }^{1, c}$, Yang Ming ${ }^{2, b}$, Li Deng-Xin ${ }^{3, c}$ \\ ${ }^{1}$ Yong-le WANG (1990-), male, master, engaged in precious metals of electronic waste \\ determination work, Donghua University,No.2999 Renmin North Songjiang District of Shanghai, \\ China \\ ${ }^{2}$ Ming YANG (1978-), Dr. Master tutor, engaged in spectral analysis, Research Center for Analysis \\ and Measurement, Donghua University, Songjiang dirsect of Shanghai, China \\ aE-mail:990478257@qq.com, byangming@dhu.edu.cn
}

Keywords:ICP-AES; Aurum; Electronic Waste; Uncertainty

Abstract:E-waste gold element content and the main source of content uncertainty is the use of inductively coupled plasma atomic emission spectrometry (ICP-AES) analysis determination. This article takes aurum as an example to analyze, compound and calculate five uncertainty components in resolving process, such as weighing samples, constant volume, standard preparation of measurement, curve fitting, and repeatability of instrument measurement to the influence of the result.

\section{Introduction}

Electronic waste contains rich in precious metals. Analysis shows that computers used in printed circuit board (PCB) generally contain precious metals (gold, silver, palladium)and non-ferrous metals (copper, zinc, iron, etc.), which have high value of recycling. E-waste contain diverse metals with different content, so the accurate determination of different types and different content of metals acquire different analysis methods. At present, common methods used for determination of trace metals and harmful elements were atomic absorption spectrometry[1],atomic emission spectrometry[2], fluorescence analysis[3], etc., These means can only be used for the detection of a single element, however, some super trace elements cannot be determined. Atomic emission spectrometry, as a modern analytical method, it has the advantages of high sensitivity, low detection limit and interference, wide linear range, etc., and under the optimal condition, it could detect multielements, simultaneously. Consequently, it is widely used in metallurgy, environment, food, agriculture and other areas of multielements analysis[4]. Especially in precious metals testing, this means shows good application prospects. All testing data have their measurement uncertainties, accordingly, and they are used for the evaluation of quantitative results, and measurement of the credibility of some parameters that can be quantitatively characterized by quantitative value dispersion[5]. At present domestic about using inductively coupled plasma atomic emission spectrometry measuring electronic waste the precious metal content of the article is less. So in this paper, the main sources of uncertainty in the determination of gold content in electronic waste were analyzed by ICP-AES based on 1059.1-2012 JJF, and then the uncertainty of measurement results were evaluated.

\section{Materials and Methods}

Instruments. The experimental apparatus used in this experiment is a Prodigy type inductively coupled plasma emission spectrometer, which is a step grating, horizontal and vertical direction observation system, the wavelength range of $165-800 \mathrm{~nm}$, high frequency generator frequency 40.68MHz. Microwave digestion instrument was purchased from Qi Yao microwave chemistry Technology Co., Ltd (Shang Hai).

Materials and Chemicals. Chip from the electronic waste, Nitric acid (HNO3, 68\%), Hydrochloric acid ( $\mathrm{HCl}, 37 \%)$, Gold standard solution(1g· L-1) was purchased from National 
Research Center for Certified Reference Material(It should be diluted stepwise before using it), DI water from a Milli-Q (Millipore) system was used in all experiments.

Containers are used in $4 \mathrm{~mol} \cdot \mathrm{L}-1 \mathrm{HNO} 3$ soaking more than $48 \mathrm{~h}$.

\section{Instrumental conditions}

\begin{tabular}{|l|l|l|l|l|l|}
\hline Power & $\begin{array}{l}\text { Cooling gas } \\
\text { flow }\end{array}$ & $\begin{array}{l}\text { auxiliary } \\
\text { gas flow }\end{array}$ & $\begin{array}{l}\text { atomizer } \\
\text { pressure }\end{array}$ & $\begin{array}{l}\text { Sample } \\
\text { lifting } \\
\text { capacity }\end{array}$ & $\begin{array}{l}\text { integral } \\
\text { time }\end{array}$ \\
\hline $1.0 \mathrm{~kW}$ & $20 \mathrm{~L} \cdot \mathrm{min}-1$ & $\begin{array}{c}0.3 \\
\mathrm{~L} \cdot \mathrm{min}-1\end{array}$ & $0.2 \mathrm{MPa}$ & $\begin{array}{l}1.2 \mathrm{~mL} \cdot \mathrm{min} \\
-1\end{array}$ & $5 \mathrm{~s}$ \\
\hline
\end{tabular}

Test method. According to GB / T 23942-2009 of the sample digestion treatment method, with electronic balance weighing a small amount of sample, then transferred to teflon digestion tank and add a certain amount of aqua regia, placed in ventilated kitchen for digestion. when complete digestion, we should use dilute nitric acid to make the volume constant. Finally, monitoring the sample by ICP-AES and then we can get the number of gold element according to the standard curve.

Mathematical model. Mathematical model used for calculating e-waste gold content is as follows. (1).

$$
\mathrm{W}=\frac{\mathrm{c}_{0} \times \mathrm{V} \times \mathrm{d}}{\mathrm{m}}
$$

Type: $W$ — The content of metal elements in samples, $\mathrm{mg} / \mathrm{kg}$;

$\mathrm{C}_{0}$ — The concentration of gold elements in digestion liquid, $\mathrm{mg} / \mathrm{L}$;

$\mathrm{V}$ _ The sample digestion liquid volume(constant volume), $\mathrm{mL}$;

d- Dilution factor;

$\mathrm{m} —$ Weighted samples amount, mg.

\section{Source of uncertainty analysis}

Factors generating uncertainty usually include experiment environment, analytical method, standard material, testing instrument and operation. The analysis method is complex and there is a certain randomness, making it difficult to quantify the uncertainty, equally. We usually use standard addition recovery experiment to decrease uncertainty. This report only analyse the uncertainties produced by the following factors:

(1)The uncertainty of scales, u1

(2)Calibration curve of nonlinear cause the uncertainty of the output value, u2

(3)The volumetric flask and pipet allow error because of volume, then cause uncertainty, u3

(4)The uncertainty of measurement repeatability, u4.

\section{The uncertainty of measurement of each weight calculation}

\subsection{The relative standard uncertainty of the sample quality, ${ }^{r e l}(\mathrm{~m})$}

\subsubsection{The uncertainty of scales, $u_{1}(k)$}

In the actual measurement process, the the sample is weighted by electronic balance, and the error of the electronic balance is propagated to the final result. According to the instrument calibration certificate, the expanded uncertainty is $\mathrm{U}=0.1 \mathrm{mg}(0 \sim 50000 \mathrm{e}), k=2$; Using class $\mathrm{B}$ to assess, it is: (2)

$$
u_{1}(k)=\frac{U}{k}=0.5 \times 10^{-4}(g)
$$


4.1.2. Scales repetitive introduce uncertainty components, $u_{1}(m)$

Weight the same sample 9 times with the balance, which has an error range of $0.0002 \mathrm{~g}$, and data showed normal distribution, $\mathrm{k}=2.97$ by looking up tables. Using class A to assess balance, it is: (3)

$$
u_{1}(m)=\frac{R}{\sqrt{n} \times k}=\frac{0.0002}{\sqrt{9} \times 2.97}=2.2 \times 10^{-5}(\mathrm{~g})
$$

4.1.3. The relative standard uncertainty of synthetic samples quality, $\quad u_{\text {rel }}(m)$

Compounding u1(k) and $\mathrm{u} 2(\mathrm{k})$ :

$$
\begin{aligned}
& u_{1}=\sqrt{u_{1}^{2}(k)+u_{1}^{2}(m)}=5.5 \times 10^{-5}(\mathrm{~g}) \\
& u_{\text {rel }}(m)=\frac{u_{1}}{m}=\frac{5.5 \times 10^{-5}}{0.5}=1.1 \times 10^{-4}
\end{aligned}
$$

\subsection{Calibration curve of nonlinear cause the uncertainty of the output value, ${ }^{r e l}$ (c)}

Gold standard solution( $\left.1 \mathrm{~g} \cdot \mathrm{L}^{-1}\right)$ was purchased from National Research Center for Standard Material with a uncertainty of $0.1 \%$. The test of samples are based of stangard curve, and the reliability of standard curve are based of standard material with certificate, $u_{2}$.

Through test, we get the standard curve, error limits for the nonlinear standard curve is described as table 1:

Table 1 The error due to nonlinear calibration curve

\begin{tabular}{lcccc}
\hline $\begin{array}{c}\text { Concentrati } \\
\text { on of standard } \\
\text { solution/ppm }\end{array}$ & $\begin{array}{c}\text { Concentration } \\
\text { of tested } \\
\text { solution/ppm }\end{array}$ & $\begin{array}{c}\text { The } \\
\text { concentration } \\
\text { difference }\end{array}$ & $\begin{array}{c}\text { Relative } \\
\text { error }(\%)\end{array}$ & $\begin{array}{c}\text { Error limits } \\
(\%)\end{array}$ \\
\hline 0.00 & 0.001 & 0.001 & 0.1 & 0.1 \\
1.00 & 0.999 & -0.001 & 0.1 & \\
10.00 & 10.000 & 0.000 & 0 & 0.000 \\
\hline
\end{tabular}

Repeat the determination eight times in the same conditions, calculation error limits each time, results are listed in table 2.

Table 2 The error limits for each group

\begin{tabular}{lllllllll}
\hline time & 1 & 2 & 3 & 4 & 5 & 6 & 7 & 8 \\
\hline $\begin{array}{l}\text { Error } \\
\text { limits }\end{array}$ & 0.1 & 0.3 & 0.1 & 0.2 & 0.3 & 0.3 & 0.1 & 0.4 \\
\hline
\end{tabular}

The above-mentioned available full width at half maximum uncertainty is $0.4 \%$, it can be considered to comply with uniform distribution. Seting coverage factor $k$ equal to $\sqrt{3}$,then component caused by the standard curve of nonlinear standard uncertainty is, $u_{\text {rel }}$ (c)

$$
\begin{aligned}
& u_{2}=\sqrt{(0.3 \%)^{2}+\left(\frac{0.4 \%}{\sqrt{3}}\right)^{2}}=0.38 \% \\
& u_{r e l}(c)=u_{2}=0.38 \%
\end{aligned}
$$

\subsection{Volume sample volume relative standard uncertainty, $u_{r e l}(V)$}

Because of the volumetric flask and pipet allow error, then they cause the uncertainty of volume, $u_{3}$.

\subsubsection{The uncertainty of constant volume, $u_{3}\left(V_{1}\right)$}

Volumetric flask has been certified, according to the calibration certificate, the error of the $50 \mathrm{~mL}$ volumetric flask is $0.05 \mathrm{~mL}$. The use of other volumetric flasks have been compared, according to the provisions in JJG 196-2006[5], a $50 \mathrm{~mL}$ volumetric flask capacity margin of error of plus or 
minus $0.05 \mathrm{~mL}$, according to the uniform distribution of class $\mathrm{B}$ assessment, $k=\sqrt{3}$, due to the volumetric flask capacity allowed error of uncertainty u3(V1) for the constant volume :

$$
u_{3}\left(V_{1}\right)=\frac{0.05}{\sqrt{3}}=0.029(\mathrm{~mL})
$$

4.3.2. Uncertainty caused by the capacity error of pipette, $u_{3}\left(V_{2}\right)$

Pipette has been tested, according to the calibration certificate, $1 \mathrm{~mL}$ single standard pipette's actual capacity is $0.999 \mathrm{~mL}$. According to the provisions in JJG 196-2006, $1 \mathrm{~mL}$ pipet's capacity margin of error is plus or minus $0.008 \mathrm{~mL}$, according to the uniform distribution of class B qualification, pipette's capacity contributes error of uncertainty for the constant volume of volume $u_{3}(\mathrm{~V} 2)$ :

$$
u_{3}\left(V_{2}\right)=\frac{0.008}{\sqrt{3}}=0.0046(\mathrm{~mL})
$$

4.3.3. The capacity volume relative standard uncertainty of the sample, $u_{\text {rel }}(V)$

Synthesis of $u_{3}\left(V_{1}\right)$ and $u_{3}\left(V_{2}\right)$ two components, and

$$
u_{\text {rel }}(V)=u_{3}=\sqrt{u_{3}^{2}\left(V_{1}\right)+u_{3}^{2}\left(V_{2}\right)}=0.0029(m L)
$$

\section{The relative standard uncertainty of testing is not repetitive, $u_{r e l}(\mathbf{w})$}

We tested the gold samples in six groups, after been diluted 10 times, they were detected ten times, consecutively. Results are tabulated in table 3.

Table 3 Repeatability determination results(n=6)

\begin{tabular}{cccccc}
\hline $\begin{array}{c}\text { Numbe } \\
\text { r }\end{array}$ & $\begin{array}{c}\text { Sample } \\
\text { quality/g }\end{array}$ & $\begin{array}{c}\text { Constant } \\
\text { volume } \\
\text { volume/ml }\end{array}$ & $\begin{array}{c}\text { Concentration of } \\
\text { gold in sample } \\
\text { solution/(mg/l) }\end{array}$ & $\begin{array}{c}\text { Gold content in } \\
\text { sample/(g/kg) }\end{array}$ & $\begin{array}{c}\text { A single set } \\
\text { of measuring } \\
\text { relative } \\
\text { standard } \\
\text { deviations/\% }\end{array}$ \\
\hline 1 & 0.5021 & 50 & 10.41162 & 10.41 & 0.59 \\
2 & 0.5034 & 50 & 10.61294 & 10.61 & 0.36 \\
3 & 0.5018 & 50 & 10.36712 & 10.36 & 0.59 \\
4 & 0.5052 & 50 & 10.75461 & 10.75 & 0.42 \\
5 & 0.5039 & 50 & 10.62243 & 10.62 & 1.21 \\
6 & 0.5025 & 50 & 10.37476 & 10.37 & 0.32 \\
\hline
\end{tabular}

As can be seen from table 3, the measured data is normally distributed, according to the class A qualification, we could calculate the relative standard deviation "s" using Bessel method[6]:

$$
s=\frac{1}{x} \sqrt{\frac{1}{n-1} \sum_{i=1}^{10}\left(x_{i}-\bar{x}\right)^{2}}
$$

The merged sample standard deviation is as follows, $s_{p}$

$$
s_{p}=\sqrt{\frac{1}{m} \sum_{i=1}^{7} s^{2}}=0.59 \%
$$

From the above can be concluded that no repetitive measurement standard uncertainty, it is

$$
u_{r e l}(w)=s_{p}=0.59 \%
$$

6. Synthesis of the calculation of relative standard uncertainty, $u_{r e l}$

Calculate the following: 


$$
u_{r e l}=\sqrt{u_{r e l}^{2}(w)+u_{r e l}^{2}(V)+u_{r e l}^{2}(m)+u_{r e l}(c)^{2}}=0.75 c
$$

\section{The expanded uncertainty}

The gold content of electronic waste was determined by calculating the average concentration of the sample: $w=10.52 \mathrm{~g} / \mathrm{kg}$, synthetic standard uncertainty: $u=u_{r e l} \times w=0.0789 \mathrm{~g} / \mathrm{kg}$, selection inclusion factors $\mathrm{k}=2$, then extended uncertainty $U=k \times u=0.16 \mathrm{~g} / \mathrm{kg}$.

The content of gold in electronic waste was determined by ICP-AES method: $w=(10.52 \pm 0.16) \mathrm{g} / \mathrm{kg}$.

\section{Conclusion}

In the whole measurement process of this experiment, the uncertainty of the determination result of the gold content in electronic waste was determined by ICP-AES method. The concentration of the standard solution, the volume of sample and the concentration of gold in the sample were determined. The uncertainty of sample weight is less than other factors, and the uncertainty of test is the main factor. To sum up, to determine the gold content of electronic waste, ICP-AES method should do the following: when you are pretreating samples, you should operate normatively to improve the veracity of monitoring results and to reduce the difference and uncertainty between samples. This time, the experiment results are valuable because they are within the margin of error and can reach the requirement.

\section{Reference}

[1] Wang Yu and Liu Xiaoduan, high resolution continuum source atomic absorption spectrometry for the determination of phosphorus in plants. Rock and mineral testing, 2009 (02): 113-118. P.

[2] Zhang Shilong, et al., inductively coupled plasma atomic emission spectrometry determination of polymetallic ores of iron, copper, lead, zinc, arsenic, antimony and molybdenum and cadmium. Physical and chemical testing (Chemical Sciences), to 2015 (07): P. 930-933.

[3] Ma Denglei, et al., fluorescence analysis method for determination of the content of total flavonoids in the leaves of Acer truncatum Bunge. Journal of Capital University of Medical Sciences, 2014 (01): P. 113-117.

[4] Zhao Yanan, Han $\mathrm{Yu}$, and Wu Jiangfeng, ICP-AES analysis of the application of research progress. Guangdong Trace Elements Science, 2010 (05): 18-24. P.

[5] Shao Wenjun, Zhang Jiguang, and Liu Jingjing, graphite furnace atomic absorption spectrometry for the determination of Trace Cadmium in rocks and soils. Rock and mineral testing, 2008 (04): 310-312. P.768-2005. JJG emission spectrometer [s] 